KAPL-P-000301

(K99056)

\title{
Spectral Ellipsometry of GaSb: Experiment and Modelling
}

\author{
G.W. Charache
}

May 1999

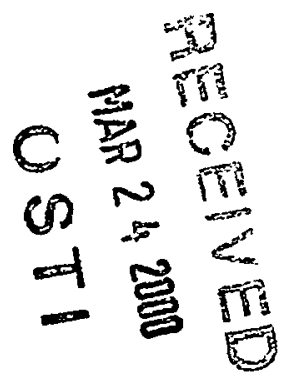

\section{NOTICE}

This report was prepared as an account of work sponsored by the United States Government. Neither the United States, nor the United States Department of Energy, nor any of their employees, nor any of their contractors, or their employees, makes any warranty, express or implied, or assumes any legal liability or responsibility for the accuracy, completeness or usefulness of any information, apparatus, product or process disclosed, or represents that its use would not infringe privately owned rights. 


\section{DISCLAIMER}

Portions of this document may be illegible in electronic image products. Images are produced from the best available original document. 
Spectral Ellipsometry of GaSb: Experiment and Modeling

M. Muñoz ${ }^{(a)}, K$. Wei and Fred H. Pollak ${ }^{(b, c)}$

Physics Department and New York State Center for Advanced Technology in Ultrafast

Photonic Materials and Applications

Brooklyn College of the City University of New York

Brooklyn, NY 11210

and

J.L. Freeouf

Interface Studies, Inc.

Katonah, NY 10536

and

G.W. Charache

Lockheed-Martin Corp.

Schenectady, NỴ 12301

The optical constants $\epsilon(\mathrm{E})\left[=\epsilon_{1}(\mathrm{E})+\mathrm{i} \epsilon_{2}(\mathrm{E})\right]$ of single crystal $\mathrm{GaSb}$ at $300 \mathrm{~K}$ have been measured using spectral ellipsometry in the range of $0.3-5.3 \mathrm{eV}$. The $\epsilon(E)$ spectra displayed distinct structures associated with critical points (CPs) at $\mathrm{E}_{0}$ (direct gap), spinorbit split $E_{0}+\Delta_{0}$ component, spin-orbit split $\left(E_{1}, E_{1}+\Delta_{1}\right)$ and $\left(E_{0}^{\prime}, E_{o}^{\prime}+\Delta_{0}^{\prime}\right)$ doublets, as well as $E_{2}$. The experimental data over the entire measured spectral range (after oxide removal) has been fit using the Holden model dielectric function [Phys. Rev. B $\underline{56}, 4037$ (1997)] based on the electronic energy-band structure near these CPs plus excitonic and band-to-band Coulomb enhancement effects at $E_{0}, E_{0}+\Delta_{0}$ and the $E_{1}, E_{1}+\Delta_{1}$ doublet. In addition to evaluating the energies of these various band-to-band CPs, information about the binding energy $\left(R_{1}\right)$ of the two-dimensional exciton related to the $E_{1}, E_{1}+\Delta_{1}$ CPs was obtained. The value of $R_{1}$ was in good agreement with effective mass $/ \vec{k} \cdot \vec{p}$ theory. The ability to evaluate $\mathrm{R}_{1}$ has important ramifications for recent first-principles band structure calculations which include exciton effects at $\mathrm{E}_{0}, \mathrm{E}_{1}$, and $\mathrm{E}_{2}$ [M. Rohlfing and S.G. Louie, Phys. Rev. Lett. 81, 2312 (1998); S. Albrecht et al, Phys. Rev. Lett, 80, 4510 (1998)]. 
Our experimental results were compared to other evaluations of the optical constants of $\mathrm{GaSb}$.

(a) Permanent address: Departamento de Fisica; CINVESTAV; Mexico DF, Mexico

(b) Also at the Graduate School and University Center of the City University of New York, New York, NY 10036

(c) E-mail address: FHPBC@CUNYVM.CUNY.EDU

PACS \# 78.20.-e, 78.20.Ci, 78.20.Bh, 78.40.Fy

GaSb.FHP 


\section{INTRODUCTION}

The semiconductor $\mathrm{GaSb}$ is of interest from both fundamental and applied perspectives. ${ }^{1}$ The spin-orbit splitting of the valence band at the center of the Brillouin zone $(B Z)$ is almost equal to the direct gap $\left(E_{0}\right)$ leading to high hole ionization effects. Among compound III-V semiconductors, $\mathrm{GaSb}$ is particularly interesting as a substrate material because its lattice parameter matches solid solutions of various ternary and quaternary III-V compounds whose band gaps cover the spectral range from $\sim 0.8-4.3$ $\mu \mathrm{m}$. Also detection at longer wavelengths, $8-14 \mu \mathrm{m}$ is possible with intersubband absorption in antimonide based superlattices. From a device point of view, GaSb-based structures have shown potential for application in laser diodes with low threshold voltage, photodetectors with high quantum efficiency, high frequency devices, superlattices with tailored optical and transport characteristics, booster cells in tandem solar cell arrangements for improved efficiency of photovoltaic cells and high efficiency thermophotovoltaic cells. ${ }^{2}$

However, in spite of its significance, relatively little work has been reported on the optical properties related to the electronic band structure. Both Aspnes and Studna ${ }^{3}$ and Zollner et $a l^{4}$ have performed spectral ellipsometry (SE) measurements of the complex dielectric function $\epsilon(E)\left[=\epsilon_{1}(E)+i \epsilon_{2}(E)\right]$ only in the range of $1.5-5.5 \mathrm{eV}$. The former investigation was at room temperature while the latter study was in the range from $10 \mathrm{~K}-740 \mathrm{~K}$. Patrini et $a f^{5}$ have published results on the optical constants at room temperature in the range of $0.0025-6 \mathrm{eV}$ using a combination of methods including reflectance, transmission, and SE (1.4-5.0 eV). Several authors have investigated the optical properties (absorption coefficient, index of refraction) in the region of the fundamental gap. ${ }^{6-11}$ Paskove $^{8}$ has presented a model for the optical constants which includes both discrete and continuum exciton contributions at $E_{0}$ but not at the $E_{1}$ critical point (transitions along the equivalent $\langle 111\rangle$ directions of the BZ). Adachi ${ }^{12}$ has modeled the optical constants (0.5-5.5 eV) using the data of Ref. 11 in the vicinity of $E_{0}$ and Ref. 3 in the range of 1.5-5.5 eV. However, his treatment does not include continuum exciton contributions, i.e., band-to-band Coulomb enhancement effects 
$(B B C E)$, at either $E_{0}$ or $E_{1}$.

In this paper we report a room temperature SE investigation of $\epsilon(\mathrm{E})$ of single crystal GaSb in the photon energy range of $0.3-5.3 \mathrm{eV}$. Distinct structures associated with critical points (CPs) at $\mathrm{E}_{0}$ (direct gap), spin-orbit split $\mathrm{E}_{0}+\Delta_{0}$ component, spin-orbit split $\left(E_{1}, E_{1}+\Delta_{1}\right)$ and $\left(E_{o}^{\prime}, E_{o}^{\prime}+\Delta_{o}^{\prime}\right)$ doublets, as well as $E_{2}$ were observed. The experimental data over the entire measured spectral range (after oxide removal) has been fit using the Holden model dielectric function ${ }^{13-15}$ based on the electronic energy-band structure near these CPs plus discrete and continuum excitonic effects at $E_{0}, E_{0}+\Delta_{0}, E_{1}$, and $E_{1}+\Delta_{1}$. The $E_{o}^{\prime}, E_{o}^{\prime}+\Delta_{o}^{\prime}$ structures were also included in the analysis. In addition to evaluating the energies of these various band-to-band CPs, it is possible to obtain information about the binding energy $\left(R_{1}\right)$ of the two-dimensional exciton related to the $E_{1}, E_{1}+\Delta_{1} C P s$. The obtained value of $R_{1}$ is in reasonable agreement with effective mass $/ \vec{k} \cdot \vec{p}$ theory. ${ }^{16}$ The ability to evaluate $R_{1}$ has important ramifications for recent first-principles band structure calculations which include exciton effects at $E_{0}, E_{1}$, and $E_{2} \cdot{ }^{17,18}$

\section{EXPERIMENTAL DETAILS}

The sample consisted of bulk GaSb (001) \{cut $4^{\circ}$ towards [110]\}. The optical data in the range of 0.75-5.3 eV [ultraviolet (UV)/visible (VIS)/near infrared (NIR)] was taken using an Instruments SA variable angle ellipsometer. For the interval of 0.3-0.8 eV [mid infrared (MIR)/far infrared (FIR)], a variable angle instrument which used a Fourier Transform Infrared Reflectometer as a light source was employed. Thus, there was some overlap between the two intervals. The UV/VIS/NIR measurements were performed with a $70^{\circ}$ incidence angle, and the MIR/FIR measurements were performed with $60^{\circ}$ and $70^{\circ}$ incidence angles. To remove the surface oxide, an etching procedure was performed on the UV/VIS/NIR ellipsometer with prealigned samples mounted vertically on a vacuum chuck in a windowless cell that maintained the surfaces in a dry nitrogen atmosphere. The details of the etching procedure are given in Ref. 13, except in this study the etch was a 3:1 mixture of $\mathrm{HCl}$ :methanol followed by a quick rinse in methanol.

IV. EXPERIMENTAL RESULTS 
Shown by the solid lines in Figs. 1(a) and 1(b) are the experimental values of the real $\left[\epsilon_{1}(E)\right]$ and imaginary $\left[\epsilon_{2}(E)\right]$ components of the complex dielectric function, respectively, as a function of photon energy, E. In the $\epsilon_{2}$ spectrum there is an absorption edge around $0.7 \mathrm{eV}$, doublet peaks in the range $2.0-2.5 \mathrm{eV}$, and a large feature with a peak around $4 \mathrm{eV}$, with some structure on the low energy side around $3.5 \mathrm{eV}$. However, in contrast to Refs. 3-5 we observe a weak feature at around $1.5 \mathrm{eV}$.

The solid lines in Figs. 2(a) and 2(b) show the experimental values of $d \epsilon_{1}(E) / d E$ and $\mathrm{d} \epsilon_{2}(\mathrm{E}) / \mathrm{dE}$, respectively, as obtained by taking the numerical derivative (with respect to E) of the solid curves in Figs. 1(a) and 1(b), respectively.

The experimental determined real $(n)$ and imaginary $(\kappa)$ components of the complex index of refraction are displayed in Figs 3(a) and 3(b), respectively, while in Fig. 4 we plot the absorption coefficient, $\alpha$. The inset of Fig. 4 shows an expanded version of $\alpha$ in the region of the direct gap.

IV. THEORETICAL MODEL

In $\mathrm{GaSb}$ the spin-orbit interaction splits the highest lying $\Gamma_{15}^{\mathrm{v}}$ valence band into $\Gamma_{8}^{\mathrm{v}}$ and $\Gamma_{7}^{\mathrm{v}}$ (splitting energy, $\Delta_{0}$ ) and the $\Gamma_{15}^{\mathrm{c}}$ conduction band into $\Gamma_{7}^{\mathrm{c}}$ and $\Gamma_{8}^{\mathrm{c}}$ (splitting energy, $\left.\Delta_{0}^{\prime}\right) .{ }^{19}$ The corresponding lowest lying transitions at $\overrightarrow{\mathrm{k}}=0$ [three-dimensional (3D) $\left.M_{0}\right]$ are labelled $E_{0}\left[\Gamma_{8}^{\mathrm{v}}\left(\Gamma_{15}^{\mathrm{v}}\right)-\Gamma_{6}^{\mathrm{c}}\left(\Gamma_{1}^{\mathrm{c}}\right)\right]$ and $E_{0}+\Delta_{0}\left[\Gamma_{7}^{\mathrm{v}}\left(\Gamma_{15}^{\mathrm{v}}\right)-\Gamma_{6}^{\mathrm{c}}\left(\Gamma_{1}^{\mathrm{c}}\right)\right]$, respectively. The spin-orbit interaction also splits the $L_{3}^{v}\left(\Lambda_{3}^{v}\right)$ valence band into $L_{4,5}^{v}\left(\Lambda_{4,5}^{v}\right)$ and $L_{6}^{v}\left(\Lambda_{6}^{v}\right)$. The corresponding $2 \mathrm{D} \mathrm{M}_{0} \mathrm{CPs}$ are designated $\mathrm{E}_{1}\left[L_{4,5}^{v}\left(L_{3}^{v}\right)-L_{6}^{c}\left(L_{1}^{c}\right)\right.$ or $\left.\Lambda_{4,5}^{v}\left(\Lambda_{3}^{v}\right)-\Lambda_{6}^{c}\left(\Lambda_{1}^{c}\right)\right]$ and $\mathrm{E}_{1}$ $+\Delta_{1}\left[L_{6}^{v}\left(L_{3}^{y}\right)-L_{6}^{c}\left(L_{1}^{c}\right)\right.$ or $\left.\Lambda_{6}^{v}\left(\Lambda_{3}^{v}\right)-\Lambda_{6}^{c}\left(\Lambda_{1}^{c}\right)\right]$, respectively. The $E_{0}^{\prime}, E_{0}^{\prime}+\Delta_{0}^{\prime}$ features correspond to transitions from the $\Gamma_{8}^{\mathrm{v}}$ valence to the spin-orbit split $\Gamma_{7}^{\mathrm{c}} / \Gamma_{8}^{\mathrm{c}}$ conduction levels and related transitions along $\langle 100\rangle .{ }^{19}$ The $E_{2}$ feature is due to transitions along $<110>(\Sigma)$ or near the X point. ${ }^{19}$

The data near the $E_{0}$ band gap were fit to a function which contains Lorentzian 
broadened (a) discrete excitonic (DE) and (b) $3 \mathrm{D} \mathrm{M}_{0}$ BBCE contributions. ${ }^{13-15}$ Both Refs. 7 and 15 demonstrate that even if the $\mathrm{E}_{0}$ exciton is not resolved, the Coulomb interaction still affects the band-to-band lineshape. Thus, $\epsilon_{2}(E)$ is given by: ${ }^{13-15}$

$$
\begin{aligned}
& \epsilon_{2}(E)=\frac{A}{E^{2}}\left\{\operatorname { I m } \left\{\left[\frac{2 R_{0}}{\left(E_{0}-R_{0}\right)-E-i \Gamma_{0}^{e x}}+\frac{2 R_{o}}{\left(E_{0}-R_{0}\right)+E+i \Gamma_{0}^{e x}}\right]+\right.\right. \\
& \left.\left.\int_{-\infty}^{\infty}\left[\frac{\theta\left(E^{\prime}-E_{0}\right)}{1-e^{-2 \pi z_{1}\left(E^{\prime}\right)}}-\frac{\theta\left(-E^{\prime}-E_{0}\right)}{1-e^{-2 \pi z_{2}\left(E^{\prime}\right)}}\right] \frac{d E^{\prime}}{E^{\prime}-E-i \Gamma_{0}}\right\}\right\}
\end{aligned}
$$

where $A$ is a constant, $E_{0}$ is the energy of the direct gap, $R_{0}$ is the effective Rydberg energy $\left[=\left(E_{0}-E_{0}^{e x}\right)\right], \Gamma_{0}^{e x}$ is the broadening of the exciton, $\Gamma_{0}$ is the broadening parameter for the band-to-band transition, $z_{1}(\mathrm{E})=\left[\mathrm{R}_{0} /\left(\mathrm{E}-\mathrm{E}_{0}\right)\right]^{1 / 2}, z_{2}(\mathrm{E})=\left[\mathrm{R}_{0} /\left(-\mathrm{E}-\mathrm{E}_{0}\right)\right]^{1 / 2}$ and $\theta(\mathrm{x})$ is the unit step function. In Eq. (1) the quantity $\mathrm{A} \propto\left(\mathrm{R}_{0}\right)^{1 / 2} \mu_{0}^{3 / 2}\left|\mathrm{P}_{0}\right|^{2}$ where $\mu_{0}$ is the reduced interband effective mass at $E_{0}$, and $P_{0}$ is the matrix element of the momentum between $\Gamma_{8}^{v}-\Gamma_{6}^{c}$. The terms in the square brackets and under the integral in Eq. (1) correspond to the $\mathrm{DE}$ and $\mathrm{BBCE}$ (continuum exciton) contributions, respectively. Since the DE is not resolved, we take $\Gamma_{0}^{\mathrm{ex}}=\Gamma_{0}$.

For the $E_{0}+\Delta_{0}$ transition a function similar to Eq. (1) was used, with $A \rightarrow B$, $\mathrm{E}_{0} \rightarrow \mathrm{E}_{0}+\Delta_{0}, \mathrm{R}_{0} \rightarrow \mathrm{R}_{\mathrm{so}}$ and $\Gamma_{0} \rightarrow \Gamma_{\mathrm{so}}$.

For the $\mathrm{E}_{1} \mathrm{CP}, \epsilon_{2}(\mathrm{E})$ is written as: ${ }^{13}$

$$
\begin{aligned}
\epsilon_{2}(E) & =\frac{C_{1}}{E^{2}}\left\{\operatorname { I m } \left\{\left[\frac{4 R_{1}}{\left(E_{1}-R_{1}\right)-E-i \Gamma_{E_{1}}}+\frac{4 R_{1}}{\left(E_{1}-R_{1}\right)+E+i \Gamma_{E_{1}}}\right]\right.\right. \\
& \left.\left.+\int_{-\infty}^{\infty}\left[\frac{\theta\left(E^{\prime}-E_{1}\right)}{1+e^{-2 \pi z_{3}\left(E^{\prime}\right)}}-\frac{\theta\left(-E^{\prime}-E_{1}\right)}{1+e^{-2 \pi z_{3}\left(E^{\prime}\right)}}\right] \frac{d E^{\prime}}{E^{\prime}-E-i \Gamma_{E_{1}}}\right\}\right\}
\end{aligned}
$$


where $C_{1}$ is a constant, $E_{1}$ is the energy of the gap, $R_{1}$ is the $2 D$ Rydberg energy, $\Gamma_{E_{1}}$ is the broadening parameter for both the exciton and band-to-band transition, $z_{3}(\mathrm{E})=$ $\left[\mathrm{R}_{1} / 4\left(\mathrm{E}-\mathrm{E}_{1}\right)\right]^{1 / 2}$, and $z_{4}(\mathrm{E})=\left[\mathrm{R}_{1} / 4\left(-\mathrm{E}-\mathrm{E}_{1}\right)\right]^{1 / 2}$.

For the $E_{1}+\Delta_{1} C P$ a function similar to Eq. (2) was used with $C_{1} \rightarrow C_{2}$ and $E_{1} \rightarrow$ $E_{1}+\Delta_{1}, \Gamma_{E_{1}} \rightarrow \Gamma_{E_{1}+\Delta_{1}}$, etc. In practice the same $2 D$ rydberg $\left(R_{1}\right)$ was used for both the $E_{1}$ and $E_{1}+\Delta_{1} C P$ features.

Reference 13 has listed relatively simple analytical expressions for $\epsilon(E)$ for $E_{0}$, $\mathrm{E}_{0}+\Delta_{0}, \mathrm{E}_{1}$, and. $\mathrm{E}_{1}+\Delta_{1}$ based on the above equations. ${ }^{20}$

The nature of the $E_{o}^{\prime}, E_{0}^{\prime}+\Delta_{0}^{\prime}$, and $E_{2}$ features is more complicated in relation to $\mathrm{E}_{0} / \mathrm{E}_{0}+\Delta_{0}$ and $\mathrm{E}_{1} / \mathrm{E}_{1}+\Delta_{1}$ since the former do not correspond to a single, well-defined CP. ${ }^{19}$ Therefore, each was described by a damped harmonic oscillator term: $:^{8,12,13}$

$$
\epsilon(E)=\frac{F(j)}{\left[1-\chi^{2}(j)\right]-i \gamma(j) \chi(j)} \quad \text { with } j=E_{o}^{\prime}, E_{0}^{\prime}+\Delta_{0}^{\prime} \text {, or } E_{2}
$$

where $F(j)$ is the amplitude, $\chi(j)=E(j) / E$, and $\gamma(j)$ is a dimensionless damping parameter.

The fact that Ref. 17 found that $E_{2}$, like the $E_{0} / E_{0}+\Delta_{0}$ and $E_{1} / E_{1}+\Delta_{1} C P$ features, contains an excitonic component provides some justification in using a damped oscillator term for this structure.

A constant, $\epsilon_{1 \infty}$, was added to the real part of the dielectric constant to account for the vacuum plus contributions from higher lying energy gaps ( $E_{1}^{\prime}$, etc. ). ${ }^{12.13}$ This quantity should not be confused with the high frequency dielectric constant $\epsilon(\infty)$.

The dotted curves in Figs. 1(a) and (b) are fits to the experimental data using the above formulas. Since the exciton at $E_{0} / E_{0}+\Delta_{0}$ has not been resolved $R_{0}(=1.6 \mathrm{meV})$ was taken from Ref. 21. Because of the large number of fitting parameters values for the various gaps and their broadening parameters were initialized from values obtained by numerically taking the first-derivative of the dielectric functions with respect to energy. 
The details of this approach are given in Refs. 13-15. The final values of the different energies are indicated by arrows in the various figures. All relevant parameters are listed in Table I. The corresponding values of $\mathrm{d} \epsilon_{1}(\mathrm{E}) / \mathrm{dE}$ and $\mathrm{d} \epsilon_{2}(\mathrm{E}) / \mathrm{dE}$, are shown by the dotted lines in Figs. 2(a) and 2(b), respectively. These were obtained from our own analytical form for the derivatives of the $E_{0} / E_{0}+\Delta_{0} C P s$, the analytical expression of Eq. (A16) of Ref. 13 for the $E_{1} / E_{1}+\Delta_{1}$ CPs, and the derivatives of Eq. (3). Overall there is very good agreement between experiment and theory for both the dielectric function [Figs. 1(a) and 1(b)] and the first derivative [Figs. 2(a) and 2(b)].

\section{DISCUSSION OF RESULTS AND SUMMARY}

The results of this experiment are in good agreement with prior studies of the optical constants of $\mathrm{GaSb} .{ }^{3-11}$ Figures $1(\mathrm{a})$ and $1(\mathrm{~b})$ correspond closely to the relevant data of Refs. 3 and 4 in the range of 1.5-5.3 eV and Ref. 4 in the range of 0.3-5.3 eV, except they have not observed $\mathrm{E}_{\mathrm{o}}^{\prime}, \mathrm{E}_{0}^{\prime}+\Delta_{0}^{\prime}$. The real component of the index of refraction $(n)$ in the vicinity of $E_{0}$, as displayed in Fig. 3(a), corresponds to the works of Paskov ${ }^{8}$ and Muñoz Uribe. ${ }^{10}$ The lineshape of our absorption coefficient in the range of $0.60-0.80 \mathrm{eV}$ (inset of Fig. 4) is similar to that presented in Ref. 7 in this region. However, our value of $\alpha \approx 10^{4} \mathrm{~cm}^{-1}$ in the plateau region above about $0.75 \mathrm{eV}$ is about a factor of two larger in relation to Ref. 7 .

Table I shows that the values of the various energy gaps obtained in this investigation, i.e., $E_{0}, E_{0}+\Delta_{0}, E_{1}-R_{1}$, $\left(E_{1}+\Delta_{1}\right)-R_{1}$, etc. are in good agreement with other selected experiments. An extensive list of these energies obtained by various methods (at different temperatures) is compiled in Ref. 4. There is some scatter in the experimental data, probably due to differences in sample quality, surface preparation and/or lineshape analysis.

The optical constants $\epsilon_{1} / \epsilon_{2}$ for $\mathrm{GaSb}^{3-5}$ as well as other diamond-and zincblendetype (DZB) semiconductors, over an extended range, have been investigated by a number of authors, ${ }^{13-15,22-27}$ mainly using SE. However, Aspnes ${ }^{3.27}$ and Cardona and co-workers ${ }^{4.23}$ did not model their results, although the latter fit derivative spectra. In Ref. 12 Adachi 
used a model in which the $E_{0}, E_{0}+\Delta_{0}, E_{1}$, and $E_{1}+\Delta_{1} C P s$ are represented by only Lorenztian broadened band-to-band single particle (BBSP) expressions, i.e., no DE. As will be discussed below the optical structure associated with the $E_{1} / E_{1}+\Delta_{1} C P$ is primarily excitonic. In later works Adachi did include DE terms but with separate amplitude factors for the DE and BBSP contributions. ${ }^{24.25}$ However, in the DE plus BBCE approach, for a given $C P$ both terms must have the same strength parameter, e.g., $A\left(E_{0}\right)$ and $C_{1}\left(E_{1}\right)$, as indicated in Eqs. (1) and (2), respectively. In Paskov's treatment ${ }^{8}$ the $\mathrm{BBCE}$ contribution is included at $\mathrm{E}_{0}$ but not $\mathrm{E}_{1}$.

The inadequacy of the BBSP approach at $E_{0}$ has been clearly demonstrated in Refs. 8 and 15. These works showed that in the region of the fundamental gap the BBCE term gave a better fit to experimental values of $\alpha$ and $\mathrm{d} \epsilon_{2} / \mathrm{dE}$, respectively, in relation to the BBSP expression. The deficiency of the BBSP model also is illustrated by Fig. 3 in Ref. 28. The fit expressions for the DE plus BBSP are considerably lower than the experimental data, particularly for the $20 \mathrm{~K}$ measurement. The effect of the BBCE term in relation to the BBSP expression is to both increase the oscillator strength and change the lineshape from a $3 \mathrm{D} \mathrm{M}_{0}$ singularity to one which approximates a $2 \mathrm{D} \mathrm{M}_{0}$ function (within about 8-10 $\mathrm{R}_{0}$ from $\mathrm{E}_{0}$ ). Kim and Sivinathan ${ }^{26}$ used DE plus BBSP terms with both Lorentzian and Gaussian broadening (increased fitting parameters). However, Aspnes $^{27}$ found no evidence for Gaussian broadening based on a Fourier analysis of the optical constants of CdTe.

Due to the relatively large values of $R_{1}(\approx 30-300 \mathrm{meV})^{13-15}$, the optical structure associated with the $E_{1}, E_{1}+\Delta_{1}$ CPS in DZB semiconductors are actually mainly the excitonic features $E_{1}-R_{1},\left(E_{1}+\Delta_{1}\right)-R_{1}$, respectively, as denoted in the figures. Almost all prior optical $1^{3-5.22-27}$ and modulated ${ }^{29.30}$ optical studies have incorrectly labelled these excitonic features as " $E_{1}, E_{1}+\Delta_{1}$ ".

Our value of $R_{1}(32 \pm 3 \mathrm{meV})$ is in good agreement with the effective mass $/ \overrightarrow{\mathrm{k}} \cdot \overrightarrow{\mathrm{p}}$ theory of Ref. 16. According to this approach:

$$
\mathrm{R}_{1} \propto \mu_{\perp} \cdot / \epsilon^{2}(\infty)
$$


where $\mu_{\perp}$ is the perpendicular reduced interband effective mass related to $E_{1}$ and $\epsilon(\infty)$ is the high frequency dieletric function. From a three band $\overrightarrow{\mathrm{k}} \cdot \overrightarrow{\mathrm{p}}$ formula the perpendicular conduction $\left(\mathrm{m}_{c, 1}^{*}\right)$ and valence $\left(\mathrm{m}_{\mathrm{v}, \perp}{ }^{*}\right)$ effective masses (in units of the free electron mass) are given by:

$$
\begin{gathered}
\frac{1}{m_{c, \perp}^{*}}=1+E_{p}\left(\frac{1}{E_{1}}+\frac{1}{E_{1}+\Delta_{1}}\right) \\
\frac{1}{m_{v, \perp}^{*}}=1-\frac{E_{p}}{E_{1}}
\end{gathered}
$$

where $E_{\mathrm{p}}$ is proportional to the square of the magnitude of the matrix element of the perpendicular momentum between the corresponding conduction and valence bands. In GaSb $\mathrm{m}_{c, \perp}^{*}=0.12$ (in units of the free electron mass) ${ }^{1}$. Hence, using the experimental values of $E_{1}$ and $E_{1}+\Delta_{1}, E_{P}$ was calculated from Eq. (4b) and $m_{v, \perp}^{*}$ was determined from Eq. (4c), to obtain $\mu_{\perp}=0.088$.

For CdTe $R_{1}=145 \mathrm{meV}$, both experimentally ${ }^{15}$ and from the considerations of Ref. 16. Therefore, using $\epsilon(\infty)=7.05$ and $\mu_{\perp}=0.15$ for $\mathrm{CdTe}^{16}$ and $\epsilon(\infty)=14.4$ for $\mathrm{GaSb}^{1}$ we find $\mathrm{R}_{\mathrm{l}}(\mathrm{GaSb}) \sim 25 \mathrm{meV}$, in reasonably good agreement with our experimental number of $32 \pm 3 \mathrm{meV}$.

The ability to measure $R_{1}$ has considerable implications for band structure calculations, both empirical ${ }^{19}$ and first principles. ${ }^{17,18}$ In the former case band structure parameters, e.g., pseudopotential form factors, are determined mainly by comparison with optical and modulated optical experiments, including the " $E_{1}, E_{1}+\Delta_{1}$ " features. Therefore, the band-to-band energies are too low by an amount $R_{1}$. Recently Rohlfing and Louie have published a first-principles calculation of the optical constants of GaAs, including excitonic effects. ${ }^{17}$ Using this formalism they have also calculated $\mathrm{R}_{0}$. Their 
approach also makes it possible to evaluate $\mathrm{R}_{1}$ from first-principles. ${ }^{31}$. Albrecht et al ${ }^{18}$ also have recently presented an $a b$ initio approach for the calculation of excitonic effects in the optical spectra of semiconductors and insulators. However, to date they have presented results for only Si.

In summary we have measured the room temperature complex dielectric function of bulk GaSb in the extended range of 0.3-5.3 eV using SE. Distinct structures related to $C P s$ associated with the direct gap, spin-orbit split $E_{0}+\Delta_{0}$, spin-orbit split $\left(E_{1}, E_{1}+\Delta_{1}\right)$ and $\left(E_{o}^{\prime}, E_{o}^{\prime}+\Delta_{o}^{\prime}\right)$ doublets, and $E_{2}$ have been observed. The $E_{0}+\Delta_{0}$ feature has not been reported in previous SE investigations. The experimental data over the entire measured spectral range has been fit using the Holden model dielectric function based on the electronic energy-band structure near these $C P$ s plus $D E$ and $B B C E$ effects at $E_{0}, E_{0}+\Delta_{0}$, $E_{1}$, and $E_{1}+\Delta_{1}$. In addition to measuring the energies of these various band-to-band CPs, we have evaluated the $2 \mathrm{D}$ exciton binding energy $R_{1}(=32 \pm 3 \mathrm{meV})$, in reasonable agreement with effective mass $/ \overrightarrow{\mathrm{k}} \cdot \overrightarrow{\mathrm{p}}$ theory. The ability to determine $\mathrm{R}_{1}$ has important ramifications for recent first-principles band structure calculations which have included excitonic effects at various critical points.

\section{ACKNOWLEDGMENTS}

The authors MM, KW, and FHP thank National Science Foundation grant \#DMR9414209, PSC/BHE grant \#666424 and the New York State Science and Technology Foundation through its Centers for Advanced Technology program for support of this project. 
Table I. Values of a number of relevant parameters obtained in this experiment. Also listed are selected values of the energy gaps, broadening parameters, etc. from other investigations.

\begin{tabular}{|c|c|c|}
\hline Parameter & $\begin{array}{c}\text { This } \\
\text { experiment }\end{array}$ & $\begin{array}{c}\text { Other selected } \\
\text { experiments }\end{array}$ \\
\hline $\mathrm{E}_{0}(\mathrm{eV})$ & $0.724 \pm 0.005$ & $\begin{array}{l}0.725^{\text {(a) }} \\
0.72^{\text {(b) }}\end{array}$ \\
\hline $\mathrm{A}\left(\mathrm{eV}^{2}\right)$ & $0.10 \pm 0.005$ & . \\
\hline $\mathrm{R}_{0}, \mathrm{R}_{\mathrm{so}}(\mathrm{meV})$ & $1.6^{(\mathrm{c}, \mathrm{d})}$ & $1.6^{(\mathrm{d})}$ \\
\hline$\Gamma_{0}, \Gamma_{0}^{\mathrm{ex}}(\mathrm{meV})$ & $15 \pm 5$ & \\
\hline $\mathrm{E}_{0}+\Delta_{0}(\mathrm{eV})$ & $1.52 \pm 0.02$ & $\begin{array}{l}1.52^{\text {(d) }} \\
1.46^{(\mathrm{b})}\end{array}$ \\
\hline $\mathrm{B}\left(\mathrm{eV}^{2}\right)$ & $0.20 \pm 0.01$ & \\
\hline$\Gamma_{s o}, \Gamma_{s o}^{e x}(m e V)$ & $15 \pm 5$ & \\
\hline$E_{1}-R_{1}(e V)$ & $2.047 \pm 0.003$ & $\begin{array}{l}2.03^{(\mathrm{e}, \mathrm{f})} \\
2.052^{(\mathrm{f}, \mathrm{g})} \\
2.05^{(\mathrm{b}, \mathrm{f})}\end{array}$ \\
\hline $\mathrm{C}_{1}\left(\mathrm{eV}^{3}\right)$ & $22.0 \pm 0.07$ & \\
\hline $\mathrm{R}_{1}(\mathrm{meV})$ & $32 \pm 3$ & \\
\hline$\Gamma_{E_{1}}, \Gamma_{E_{1}}^{e x}(m e V)$ & $95 \pm 10$ & $60^{(n)}$ \\
\hline$\left(E_{1}+\Delta_{1}\right)-R_{1}(e V)$ & $2.488 \pm 0.005$ & $\begin{array}{l}2.49^{(\mathrm{e}, \mathrm{h})} \\
2.494^{(\mathrm{g}, \mathrm{h})} \\
2.50^{(\mathrm{b}, \mathrm{h})}\end{array}$ \\
\hline
\end{tabular}




\begin{tabular}{|c|c|c|}
\hline$\Gamma_{E_{1}+\Delta_{1}}, \Gamma_{E_{1}+\Delta_{1}}^{\text {ex }}$ & $220 \pm 10$ & $70^{(\mathrm{s})}$ \\
\hline $\mathrm{C}_{2}\left(\mathrm{eV}^{2}\right)$ & $30.0 \pm 0.01$ & \\
\hline $\mathrm{E}_{0}^{\prime}(\mathrm{eV})$ & $3.40 \pm 0.01$ & $3.27^{(\mathrm{e})}$ \\
\hline $\mathrm{F}\left(\mathrm{E}_{0}^{\prime}\right)$ & $1.20 \pm 0.01$ & \\
\hline$\gamma\left(E_{0}^{\prime}\right)$ & $230 \pm 10$ & \\
\hline $\mathrm{E}_{\mathrm{o}}^{\prime}+\Delta_{0}^{\prime}(\mathrm{eV})$ & $3.79 \pm 0.02$ & $3.56^{(e)}$ \\
\hline $\mathrm{F}\left(\mathrm{E}_{0}^{\prime}+\Delta_{0}^{\prime}\right)$ & $1.03 \pm 0.01$ & \\
\hline$\gamma\left(E_{o}^{\prime}+\Delta_{0}^{\prime}\right)$ & $170 \pm 10$ & \\
\hline$E_{2}(e V)$ & $4.100 \pm 0.005$ & $\begin{array}{l}4.20^{(e)} \\
4.20^{(g)} \\
4.0^{(b)}\end{array}$ \\
\hline $\mathrm{F}\left(\mathrm{E}_{2}\right)$ & $1.87 \pm 0.01$ & $5.69^{(b)}$ \\
\hline$\gamma\left(\mathrm{E}_{2}\right)$ & $125 \pm 10$ & $290^{(b)}$ \\
\hline$\epsilon_{1 \infty}$ & 1.97 & $1.0^{(b)}$ \\
\hline
\end{tabular}
(a) Ref. 1 .
(b) Ref. 12 .
(c) Fixed.
(d) Ref. 21.
(e) Ref. 29.
(f) Incorrectly labelled $E_{1}$.
(g) Ref. 4. 
(h) Incorrectly labelled $E_{1}+\Delta_{1}$.

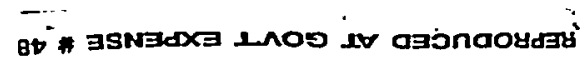




\section{REFERENCES}

1. P.S. Dutta and H.L. Bhat, J. Appl. Phys. 81, 5821 (1997).

2. G.W. Charache, J.L. Egley, D.M. Depoy, L.R. Danielson, M.J. Freeman, R.J. Dziendziel, J.F. Moynihan, P.F. Baldasaro, B.C. Campbell, C.A. Wang, H.K. Choi, G.W. Turner, S.J. Wojtczuk, P. Colter, P. Sharps, M. Timmons, R.E. Fahey, and K. Zhang, J. Electron. Mat. 27, 1038 (1998).

3. D.E. Aspnes and A.A. Studna, Phys. Rev. B 27, 985 (1983).

4. S. Zollner, M. Garriga, J. Humlicek. S. Gopalan, and M. Cardona, Phys. Rev. B 43, 4349 (1991).

5. M. Patrini, G. Guizzei, M. Galli, R. Ferrini, A. Bosacchi, S. Franchi, and R. Magnanini, Solid State Comm. 101, 93 (1997).

6. Z. Bachan, E. Kierzek-Pecold, and J. Kolodziejczak, Phys. Stat. Sol. 42, K101 (1970).

7. P.P. Paskove, IEEE J. Quant. Electronics 30, 2771 (1994).

8. P.P. Paskove, J. Appl. Phys. 81, 1890 (1997).

9. C. Ghezzi, R. Magnanini, A. Parisini, B. Rotelli, L. Tarricone, A. Bosacci, and S. Franchi, Semicond. Sci. Technol. 12, 858 (1997).

10. M. Muñoz Uribe, R.S. Miranda, M.B. Zakia, C.F. de Souza, C.A. Riberio, J.H. Clerice, and N.B. Patel, Mat. Sci. and Engin. B 38, 259 (1996).

11. B.O. Seraphin and H.E. Bennett, in Semiconductors and Semimetals, Vol. 3, edited by R.K. Wilardson and A.C. Beer (Academic, New York, 1967) p. 499.

12. S. Adachi, J. Appl. Phys. 66, 6030 (1989).

13. T. Holden, P. Ram, F.H. Pollak, J.L. Freeouf, B.X. Yang, and M.C. Tamargo, Phys. Rev. B 56, 4037 (1997).

14. T. Holden, F.H. Pollak, J.L. Freeouf, G.W. Charache, J.E. Raynolds, and C. Geller, Proc. 4th NREL Thermophotovoltaic Generation of Electricity Conference, Denver, 1998, ed. T.J. Coutts, J. P. Brenner, and C.S. Allman, AIP Conf. Proc. 460 (American Institute of Physics, Woodbury, 1999), p. 39.

15. K. Wei, F.H. Pollak, J.L. Freeouf, D. Shvydka, and A.D. Compaan, "Optical Properties of $\mathrm{CdTe}_{1-x} \mathrm{~S}_{x}(0 \leq x \leq 1)$ : Experiment and Modeling", to be published in $\mathrm{J}$. Appl. Phys.

16. Y. Petroff and M. Balkanski, Phys. Rev. B 3, 3299 (1971). 
17. M. Rohlfing and S.G. Louie, Phys. Rev. Lett. 81, 2312 (1998)

18. S. Albrecht, L. Reining, R. Del Sole, and G. Onida, Phys. Rev. Lett, 80, 4510 (1998).

19. M.L. Cohen and J.R. Chelikowsky, in Electronic Structure and Optical Properties of Semiconductors (Springer, Berlin, 1989).

20. Equation (A13) in Ref. 13 should read $\xi^{2}(X)=4\left(E_{1}-X\right) / G$

21. Landolt-Börnstein-Numerical Data and Functional Relationships in Science and Technology, ed. by O. Madelung, M. Schultz, and H. Weiss, Vols. 17a and 17b (Springer, New York, 1982).

22. F.H. Pollak in 1967 International Conference on the II-VI Semiconducting Compounds, ed. by D.G. Thomas (V.A. Benjaminn New York, 1967) p. 552.

23. P. Lautenschlager, M. Garriga, S. Logothetidis, and M. Cardona, Phys. Rev. B 35, 9174 (1987); P. Lautenschlager, M. Garriga, and M. Cardona, Phys. Rev. B 36, 4813 (1987).

24. S. Adachi, T. Kimura, and N. Suzuki, J. Appl, Phys. 74, 3435 (1993); S. Adachi and T. Taguchi, Phys. Rev. B 43, 9569 (1991).

25. K. Suzuki and S. Adachi, J. Appl. Phys. 83, 1018 (1998).

26. C.C. Kim and S. Sivinathan, J. Appl. Phys. 78, 4003 (1995); alos, Phys. Rev. B 53, 1475 (1996).

27. D.E. Aspnes, Solar Energy Materials and Solar Cells 32, 413 (1994).

28. S. Adachi, Phys. Rev. B 41, 1003 (1990).

29. M. Cardona in Modulation Spectroscopy (Academic, New York, 1969).

30. F.H. Pollak and H. Shen, Mater. Sci. and Engineering R 10, 275 (1993).

31. S. Louie, private communication. 


\section{FIGURE CAPTIONS}

Fig. 1 The solid and dashed lines are the experimental and fit values, respectively, of the (a) real $\left(\epsilon_{1}\right)$ and (b) imaginary $\left(\epsilon_{2}\right)$ components of the complex dielectric function of $\mathrm{GaSb}$.

Fig. 2 The solid and dashed lines are the experimental and fit values, respectively, of (a) $d \epsilon_{1} / d E$ and (b) $d \epsilon_{2} / d E$ of GaSb.

Fig. 3 The solid lines are the experimental values of the (a) real (n) and (b) imaginary ( $\kappa$ ) components of the complex index of refraction of $\mathrm{GaSb}$.

Fig. 4 The solid line is the experimental value of the absorption coefficient, $\alpha$, of GaSb. The inset shows an expanded version in the region near $E_{0}$. 


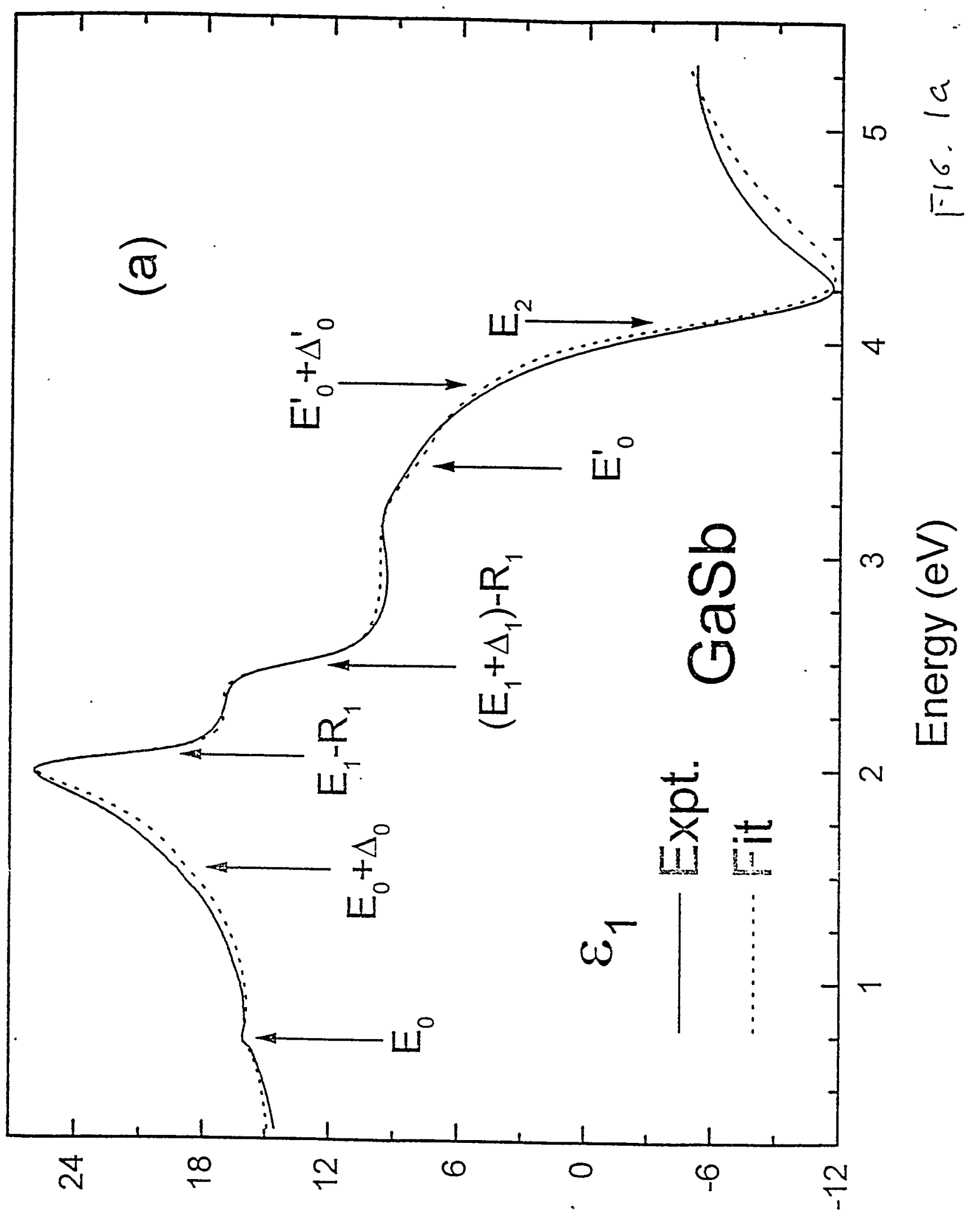




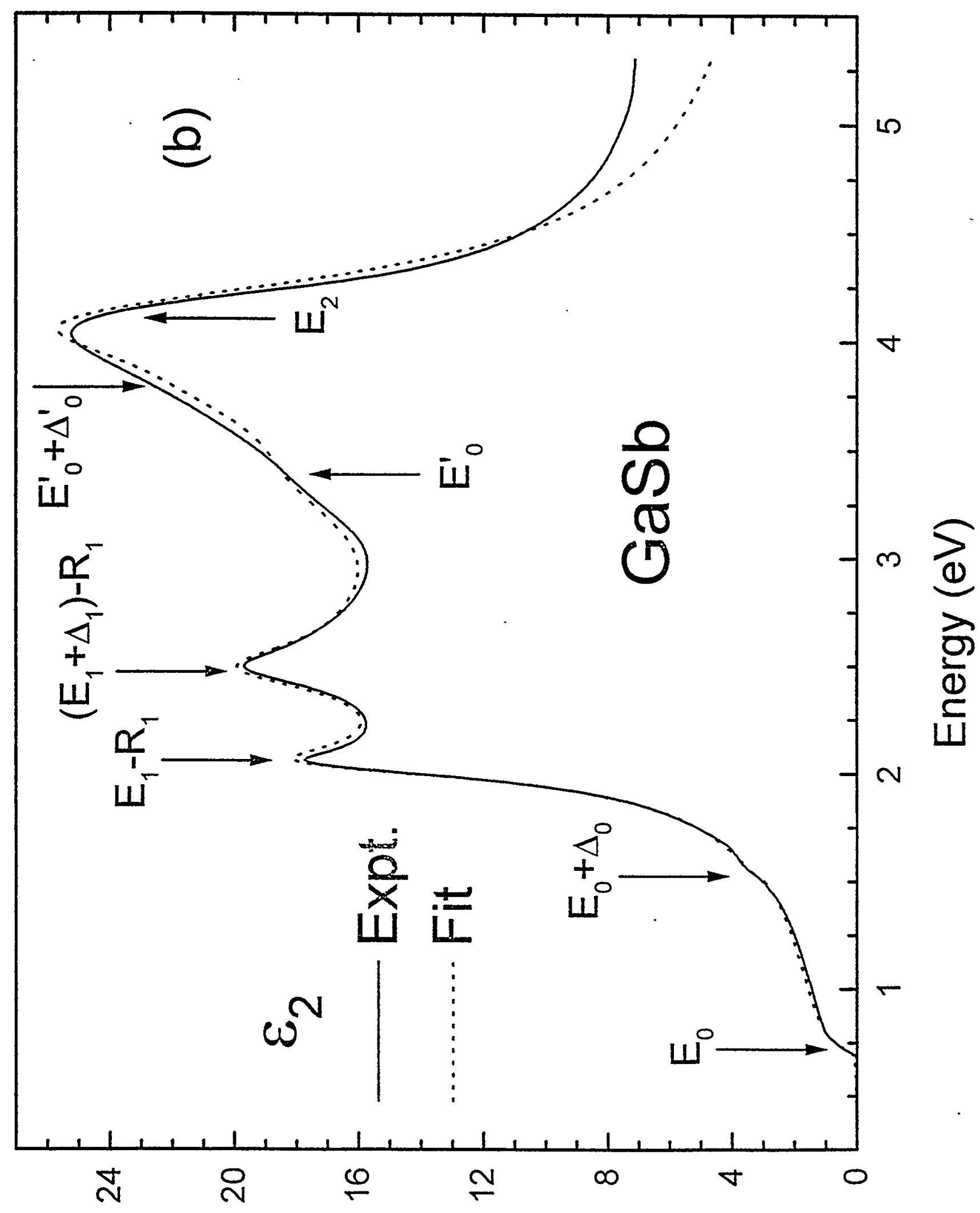




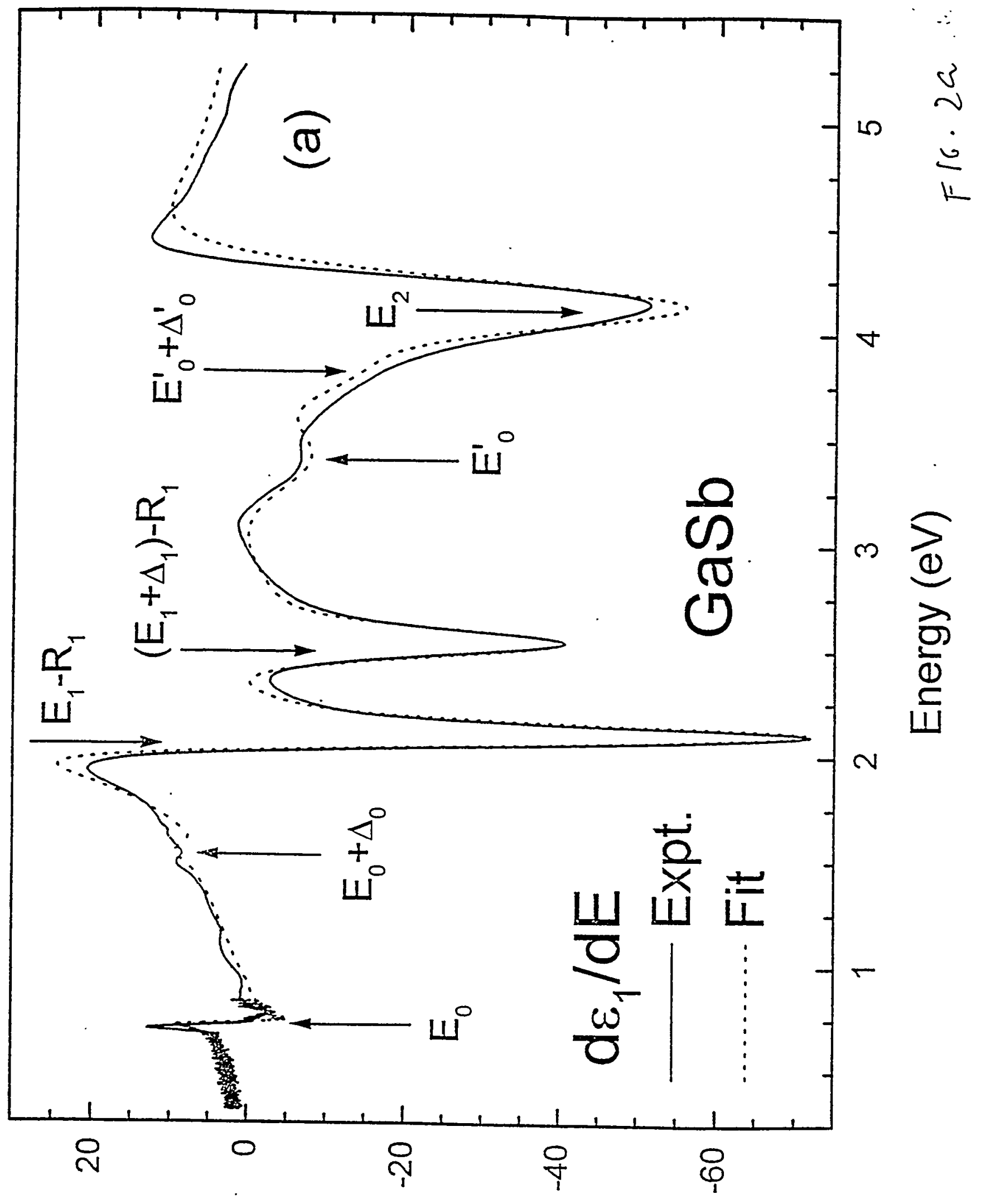




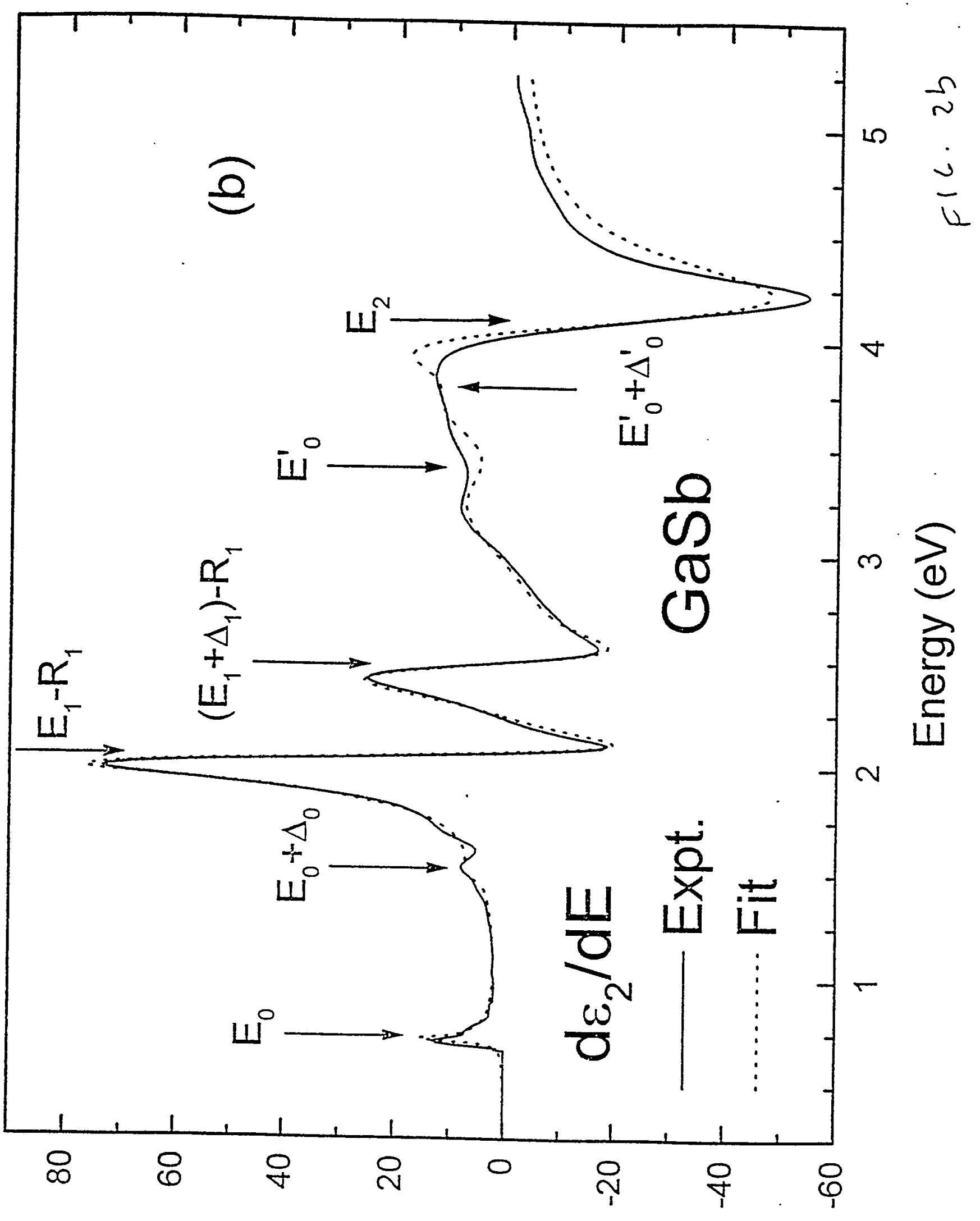




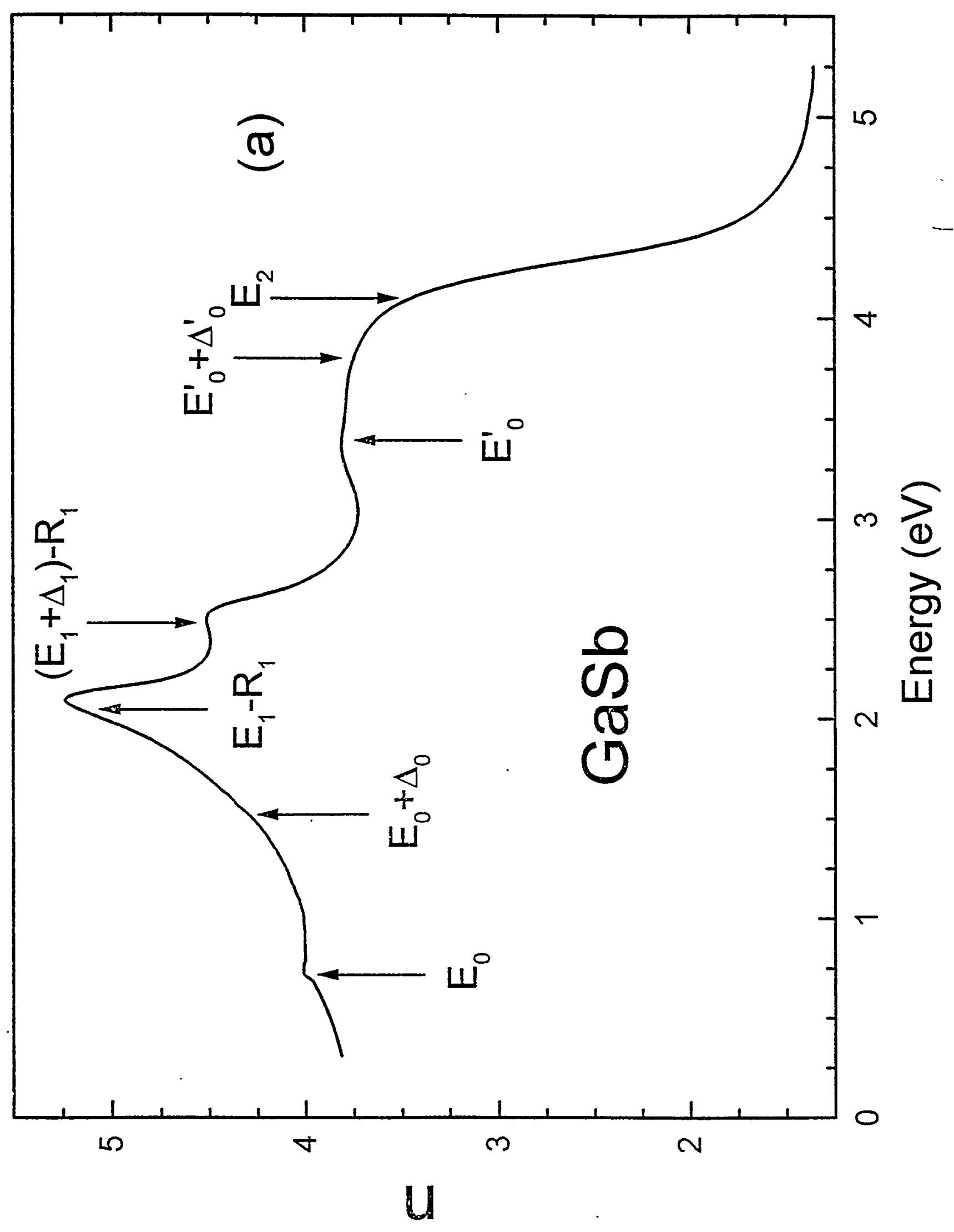

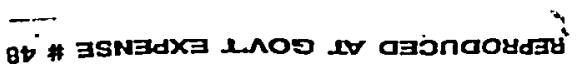




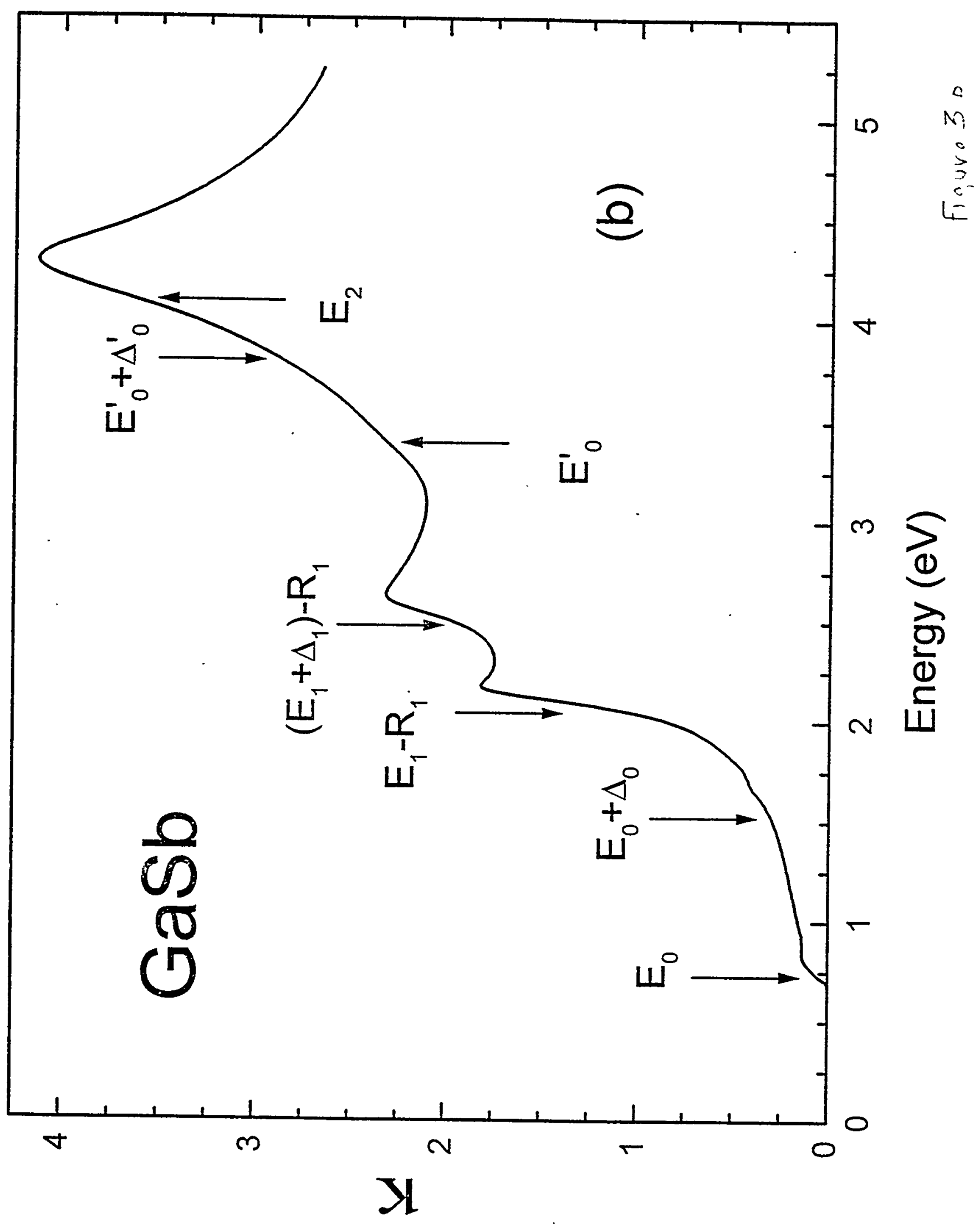




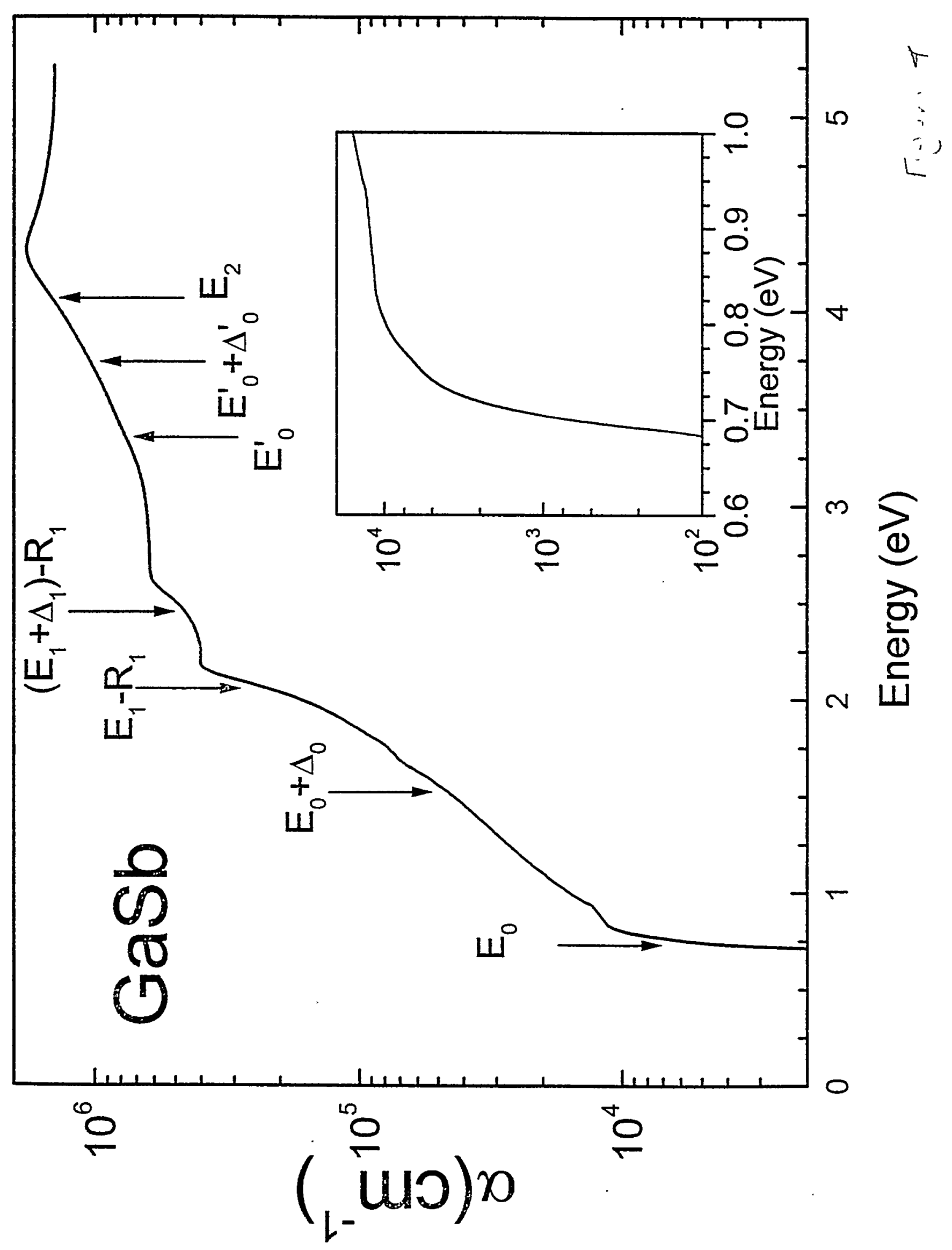

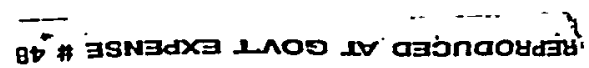

